Anal ysi s of the three- di mensi onal traj ect or i es of dust s observed wi th a ster eoscopi c fast framing camera in the Large Hel i cal Devi ce

\begin{tabular}{|l|l|}
\hline $\begin{array}{l}\text { jour nal or } \\
\text { publ i cat i on ti t l e }\end{array}$ & Jour nal of Nucl ear Nat er i al s \\
\hline vol une & 463 \\
\hline page r ange & $861-864$ \\
\hline year & $2015-08$ \\
\hline URL & ht t p: //doi . or g/10. 15047/00012489 \\
\hline
\end{tabular}




\title{
Analysis of the three-dimensional trajectories of dusts observed with a stereoscopic fast framing camera in the Large Helical Device
}

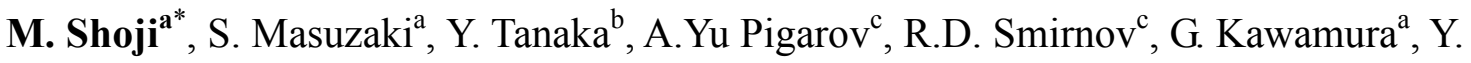 \\ Uesugi $^{\mathrm{a}}$, H. Yamada ${ }^{\mathrm{a}}$ and the LHD Experiment Group ${ }^{\mathrm{a}}$ \\ ${ }^{a}$ National Institute for Fusion Science, 322-6 Oroshi-cho, Toki 509-5292, Gifu, Japan \\ ${ }^{\mathrm{b}}$ Kanazawa University, Kakuma, Kanazawa 920-1192, Japan \\ ${ }^{\mathrm{c}}$ University of California at San Diego, La Jolla, CA 92093, USA
}

\begin{abstract}
The three-dimensional trajectories of dusts have been observed with two stereoscopic fast framing cameras installed in upper and outer viewports in the Large Helical Device (LHD). It shows that the dust trajectories locate in divertor legs and an ergodic layer around the main plasma confinement region. While it is found that most of the dusts approximately move along the magnetic field lines with acceleration, there are some dusts which have sharply curved trajectories crossing over the magnetic field lines. A dust transport simulation code was modified to investigate the dust trajectories in fully three dimensional geometries such as LHD plasmas. It can explain the general trend of most of observed dust trajectories by the effect of the plasma flow in the peripheral plasma. However, the behavior of the some dusts with sharply curved trajectories is not consistent with the simulations.
\end{abstract}

PACS: $52.55 . \mathrm{Hc}, 52.65 . \mathrm{Cc}, 52.70 . \mathrm{Kz}, 52.70 . \mathrm{Nc}$

PSI-21 Keywords: Visible imaging, Dust, LHD, EMC3, EIRENE

*Corresponding Author Address: National Institute for Fusion Science, 322-6 Oroshi-cho,

Toki 509-5292, Japan

*Corresponding Author e-mail: shoji@LHD.nifs.ac.jp

Presenting Author: Mamoru Shoji

Presenting Author e-mail: shoji@LHD.nifs.ac.jp 


\section{Introduction}

Dusts in plasma confinement devices have recently gained attention from the viewpoint of the influence on the main plasma performances [1,2]. Deposition layers including impurities accumulated in the divertor region can be exfoliated and become dust particles [3]. It is concerned that the impurities included in the dusts penetrating into the main plasma induce radiation collapses during plasma discharges.

Recent ICRF heated long pulse discharges in the Large Helical Device (LHD) have been terminated with abrupt increase of carbon emission. A fast framing camera for monitoring plasmas observed the release of a large amount of dusts from a divertor region, suggesting that the penetration of dusts into the main plasma caused the radiation collapse at the end of the discharges. It strongly motivated us to study transport of dusts. In order to investigate the dust transport, measurement of the three-dimensional trajectories of dusts is indispensable. Meanwhile, dust transport simulation has been developing in the last more than ten years [4]. Analysis using a dust transport simulation code is useful for understanding the physical mechanisms of the dust trajectories.

In this paper, the following two experimental and numerical results are presented:

1. Observations of the three-dimensional trajectories of dusts with two stereoscopic fast faming cameras installed in two different positions (upper and outer ports),

2. Simulations by a dust transport code which is modified to be applied to fully three-dimensional configurations such as LHD plasma geometries.

In the last section, the observed dust trajectories are investigated using the modified dust transport simulations code, which is the first trial in non-axisymmetric helical plasma confinement devices.

\section{Experimental setup for stereoscopic measurement of dust trajectories}

The LHD is the largest helical device with superconducting helical and poloidal coils 
for plasma confinement, which forms toroidally twisted elliptical plasmas in the vacuum vessel [5]. One of the features of the LHD is the three-dimensionally complicated magnetic field line structures in the peripheral plasma, which are mainly composed of two parts: an ergodic layer and divertor legs. In the ergodic layer, magnetic field lines are ergodized around the Last Closed Flux Surface (LCFS). Four bundled magnetic field lines (the divertor legs) are deviated from two X-points in the outer edge of the ergodic layer, which directly connect the magnetic field lines to the vacuum vessel (stainless steel) and divertor plates (isotropic carbon graphite). The divertor plates are installed along the four lines of the strike points which are helically distributed on the surface of the vacuum vessel.

As shown in Figure 1 (a), two fast framing cameras have been equipped in an outer port (3-O) and an upper port (7.5-U) with stereo optics by which two images are combined into one image. Two optical lenses have been set at two different positions in these ports, one of which is separated from other one at a distance of about $1 \mathrm{~m}$. The lenses are connected to the stereo optics via image fibers, which enables one camera to acquire two images viewed from two different positions simultaneously. In order to know the exact spatial configuration of the cameras, several benchmarks were set on the surface of some components in the vacuum vessel, which locate in the viewing area of the cameras. The three-dimensional positions of the benchmarks were measured on the site after the experimental campaigns.

The fields of view of the two stereoscopic fast framing cameras in the upper and outer ports are illustrated in Figure 1 (b) and (c), respectively. The camera installed in the upper port can stereoscopically observe the upper and lower divertor regions and divertor plates at a toroidal position where the plasma is vertically elongated. The viewing area from the outer port can stereoscopically observe the peripheral plasma in the outboard side of the torus at a toroidal position where the plasma is horizontally elongated. 


\section{Observations of dust trajectories with stereoscopic fast framing cameras}

Stereoscopic observations of six representative dust trajectories taken with the fast framing cameras installed in the upper and outer ports are shown in Figure 2 (a) and (b), respectively. These figures are superimposed images taken at some different times and plasma discharges in the case of an inward magnetic axis shift configuration (the radial position of the magnetic axis: $\left.R_{\mathrm{ax}}=3.60 \mathrm{~m}\right)$. Dusts in the plasma are identified as small incandescent moving spots on the images. One to one correspondence of a dust on the stereoscopic image is possible by the different time trends of the intensity, velocity, shape and trajectory of incandescent spots as long as the number of the spots is less than several ones in the image. The arrows and small triangles in the figures indicate the direction of the movement of the dusts, and the positions at the intervals of the exposure time of the cameras. It clearly presents that the dusts move with acceleration and there are many varieties of dust trajectories: for example, almost straight lines, sharply curved ones and trajectories with different moving directions, etc.

\section{Reconstruction of the three-dimensional trajectories of dusts}

The three-dimensional trajectories of dusts are reconstructed by analyzing the traces of the incandescent moving spots on the stereoscopic images observed from the two different positions using a pinhole camera model. It requires a camera matrix which is for mapping three-dimensional coordinates of a dust position to the two-dimensional coordinates on the image sensor in the fast framing cameras. In order to obtain the matrix, spatial calibration of the cameras was performed after the experimental campaigns by reproducing the spatial configuration of the optics in a laboratory using the information of the three-dimensional positions of the benchmarks.

Figure 3 (a) shows observations of the three-dimensional trajectories of dusts reconstructed by the above method. Colored lines (dots) represent the dust positions observed 
at the reduced intervals of the exposure time of the fast cameras. In this figure, the dust trajectories observed from the two different toroidal positions (3-O and 7.5-U) are combined into one toroidal section $\left(0^{\circ}<\phi<36^{\circ}\right)$ in the LHD. Small white and red dots express the magnetic field lines in the peripheral plasma and those on the LCFS, respectively. It shows that the dusts locate in the ergodic layer and in the lower and upper divertor legs. Enlarged views of the observed dust trajectories from the upper and outer ports are presented in Figure 3 (b) and (c), respectively. The six representative dust trajectories shown in Figure 2 are indicated by white arrows, and the moving directions are expressed by small black arrows. Figure 3 shows that most of the dusts approximately move along the magnetic field lines with acceleration. Dust trajectories in the upper and lower divertor legs, each of which is aggregate of colored lines (dots) in left-lower and right-upper area in Figure 3 (b), seem to show that the dusts are brushed away along the magnetic field lines in both of the divertor legs.

The effects of the peripheral plasma on the dust transport have been already reported by an analysis using a dust transport simulation code (DUSTT) in two-dimensional Tokamak plasmas [6-8]. The simulation shows that the ion drag force due to the plasma flow along the magnetic field lines is the dominant force on the motion of dusts in the peripheral plasma. The above observations experimentally support the validity of the dust transport simulation code. However, it should be noted that there are some dust trajectories crossing over the magnetic field lines with sharply curved trajectories in both the ergodic layer and the divertor legs.

\section{Simulation analysis of three-dimensional dust trajectories}

The dust transport simulation code has been applied to the analysis of the three-dimensional dust trajectories in the LHD peripheral plasma. This code calculate the equation of motion of a spherical shaped dust coupled with the heat, charge, mass equations of the dust in plasmas with including the effect of the ion/neutral drag force, gravity force, electrostatic force and dust ablation processes until the dusts are fully evaporated or 
sublimated [9]. It has been originally developed for analysis in axisymmetric configurations like Tokamak plasmas. In order to apply the simulation code to non-axisymmetric configurations such as LHD plasmas, a dust tracking sub-program in the simulation code was implemented in a three-dimensional neutral particle transport simulation code (EIRENE) [10]. This modification treats the dusts as special test particles, which physical properties are based on the DUSTT code, in the particle transport simulation in the EIRENE code. It enables dust transport simulation in fully three-dimensional geometries using the grid model for the EIRENE code.

The observations of the six representative dust trajectories shown in Figure 2 are analyzed using the modified dust transport simulation code. The dust trajectories are calculated from the observed initial positions obtained by the above reconstruction method. The initial velocities and moving directions of the dusts are derived from the reconstructed three-dimensional trajectories. The background plasma parameter profiles (the electron/ion temperature and density, and the parallel plasma flow velocity, etc.) were calculated by the EMC3-EIRENE code $[11,12]$. In this simulation, the dusts are assumed to be composed of carbon, because it has been experimentally found that carbon is the major composition of the dusts collected in the vacuum vessel [13].

Figure 4 (a) gives a three-dimensional vector plot of the calculated parallel plasma flow density with indication of the toroidal direction by small black and white arrows, showing the three-dimensionally complicated flow density distribution in the peripheral plasma. The calculated trajectories of the six representative dusts are shown by colored lines (dots). Figure 4 (b) and (c) are the enlarged views of the calculated dust trajectories, which are seen from the positions of the upper and outer ports, respectively. The direction of the plasma flow around the positions of the dusts in the plasma periphery is expressed as black and white arrows. It shows that the calculated dust trajectories for some different dust radii which are in the range of those of collected dusts in the vacuum vessel after experimental campaigns [13], 
because the dust radius is a free parameter in the simulation. The observed dust trajectories \#1 and \#2 shown in Figure 3 (b), and \#4 and \#5 in (c) are within the range of the simulations calculated by changing the dust radius. The observations of the dust moving velocities are also within the range of the simulations.

In calculated dust trajectories shown in Figure $4(\mathrm{~b})$, relatively small sized dusts tend to move along the magnetic field lines by the effect of the plasma flow in the upper and the lower divertor legs in which the dusts are swept away to the outside of the plasma or are immediately sublimated. Relatively larger sized dusts show a tendency to keep the initial directions due to the large inertial effect. In the trajectories of dusts \#5 and \#6 in Figure 4 (c), the initial direction of the dusts is approximately consistent with that of the plasma flow in the ergodic layer. The simulation shows that the length of the trajectories (life time) of the dusts strongly depends on the size of the dusts. In the trajectories of dust \#6, dusts with radii more than $4 \mu \mathrm{m}$ are swept away to the outside of the plasma through the ergodic layer by the large inertial effect. The trajectory of dusts \#4 is somewhat complicated, showing that the initial dust position is in the region where the direction of the plasma flow is to the upper-left side and the plasma flow density is relatively small. In the initial phase, the trajectory of the small sized dust $\left(r_{\text {dust }}=10 \mu \mathrm{m}\right)$ change the direction by the effect of the plasma flow and it finally sublimates in the ergodic layer. The relatively large sized dusts can move to the radially outer region of the ergodic layer because of the larger inertial effects and the longer life times. The dusts reaching to the outer region near an outer divertor leg are transported to the lower-right side by the effect of the strong plasma flow close to the divertor leg.

Being different from the trajectories of dusts \#1, \#2 in Figure 4 (b), and \#4, \#5 in Figure 4 (c), the observations of the sharply curved dust trajectories indicated as \#3 in Figure 3 (b) and \#6 in Figure (c) cannot be explained by the dust transport simulation. It strongly suggests that physical mechanisms not included in the simulation code have to be considered. A kind of non-asymmetric rocket force induced by ablation of an element included in 
non-spherical shaped mixed-material dusts may be a possible mechanism for the discrepancy. Non-thermal high energy ions produced by NBI/ICRFs in the peripheral plasma can also be a candidate for it. In addition to that, the assumption adopted in the present simulation code should be reexamined for detailed analysis of individual dust transport in plasmas.

\section{Summary}

The trajectories of dusts have been observed with two stereoscopic fast framing cameras installed in outer and upper ports. It shows that dust trajectories locate in the ergodic layer and in the divertor legs. While the observations show that most of the dusts approximately move along the magnetic field lines in the peripheral plasma, there are some dusts radially crossing over the magnetic field lines with sharply curved trajectories. A dust transport simulation code, which is modified to the analysis in fully three-dimensional geometries, demonstrates that the simulation can explain the general trend of most of dust trajectories by the effect of the plasma flow. However, some dust trajectories crossing over the magnetic field lines are not consistent with the simulations.

\section{Acknowledgements}

The authors would like to thank all the members of the LHD, and they are grateful for the support for the computational resources of the LHD numerical analysis server, which is supported by the NIFS budget code NIFS12KNVN236. This work is financially supported by NIFSULPP015 and by NIFS/NINS under the project of Formation of International Scientific Base and Network. It is performed with the support and under the auspices of the NIFS Collaboration Research program NIFS13KLPP026. 


\section{References}

[1] S. I. Krasheninnikov et al., Plasma Phys. Control Fusion 53 (2011) 083001.

[2] S.Ratynskaia et al., Plasma Phys. Control. Fusion 53 (2011) 074009.

[3] M. Tokitani et al., J. Nucl. Mater. 438 (2013) S818.

[4] S. I. Krasheninnikov et al., Phys. Plasmas 11 (2004) 3141.

[5] A. Komori et al., Fusion Sci and Technol. 58 (10) (2010) 1.

[6] A. Yu Pigarov et al., J. Nucl. Mater. 363-365 (2007) 216.

[7] R. D. Smirnov et al., Plasma Phys. Control Fusion 49 (2007) 347.

[8] Y. Tanaka et al., J. Nucl. Mater. 415 (2011) S1106.

[9] A. Yu Pigarov et al., Phys. Plasmas 12 (2005) 122508.

[10] D. Reiter et al., Fusion Sci. Technol. 47 (2005) 172.

[11] Y. Feng et al., Plasma Phys. Control. Fusion 44 (2002) 611.

[12] G. Kawamura et al., Contrib. Plasma Phys. 54 (2014) 437.

[13] K. Koga et al., in: Proc. 24th IAEA Fusion Energy Conf., San Diego, 2012, EX/P5-32. 


\section{Figure captions}

Fig. 1. The top view of the experimental setup of the stereoscopic fast framing cameras for three-dimensional dust trajectory measurements (a). The fields of view of the stereoscopic fast framing cameras installed in an upper port (7.5-U) (b) and an outer port (3-O) (c).

Fig. 2. Stereoscopic observations of six representative dust trajectories taken with the fast framing camera installed in the upper (a) and the outer ports (b).

Fig. 3. A view of the reconstructed three-dimensional dust trajectories observed from the upper and outer ports (colored lines) (a). In this figure, the magnetic field lines in the plasma periphery and those on the last closed flux surface are plotted by small white and red dots, respectively. The enlarged images of the dust trajectories viewed from the upper (b) and outer ports (c). The magnetic field lines in the peripheral plasma are represented as small white dots. The trajectories of the six representative dusts shown in Figure 2 are indicated by thick white arrows, where the observed dust trajectories are shown by a series of colored dots which positions are observations taken at intervals of the exposure time of the fast framing cameras.

Fig. 4. A three-dimensional vector plot of the plasma flow density calculated by the EMC3-EIRENE code. The simulations of the three-dimensional trajectories of the six representative dusts are shown by colored lines (dots) with indications of the initial positions (open yellow circles) (a). Enlarged views of the calculated dust trajectories seen from the upper (b) and outer ports (c) in the case of some different dust radii. The positions where the dusts are fully sublimated are marked by colored X signs. The magnitude and directions of the plasma flow density are expressed as small white and black arrows. The arrows in the left and right side in Figure 4 (b) express the plasma flow density in the upper and lower side of the peripheral plasma, respectively. The arrows in Figure 4 (c) are the plasma flow density in the 
ergodic layer in the outboard side of the torus. 
Figures

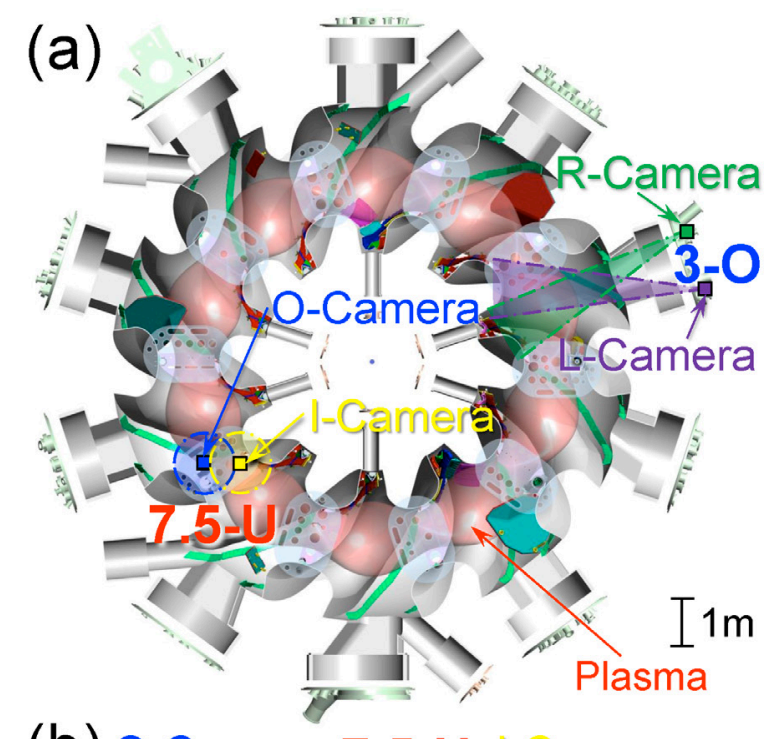

(b) O-Camera 7.5-U I-Camera

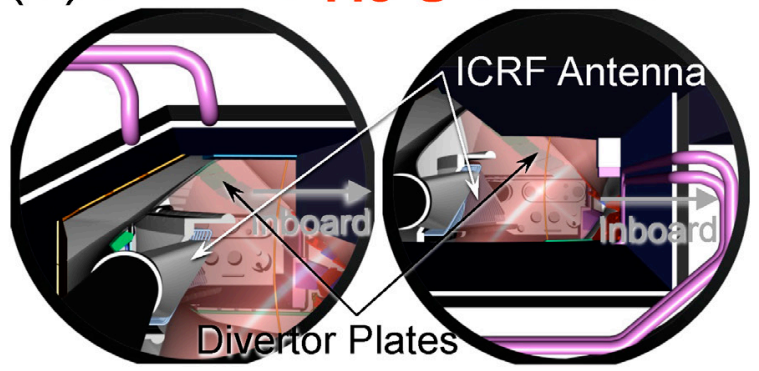

(c) L-Camera 3-O R-Camera

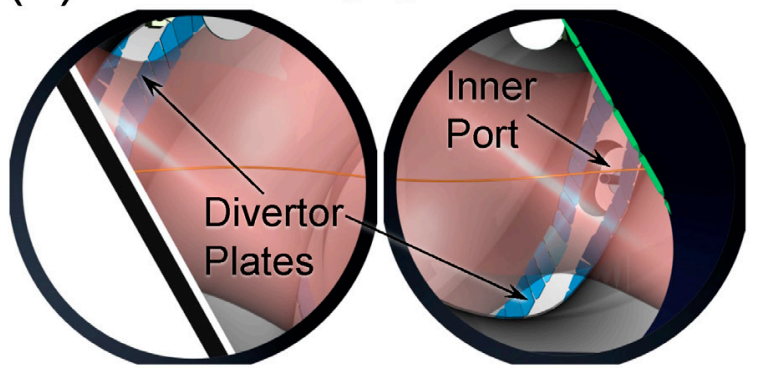

Figure 1 
(a) O-Camera

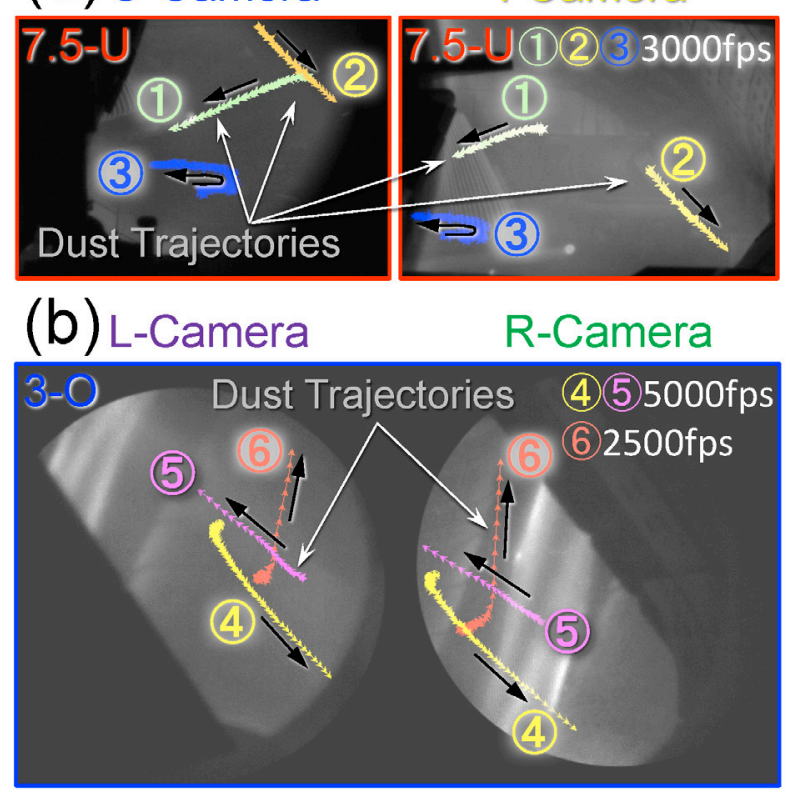

Figure 2 

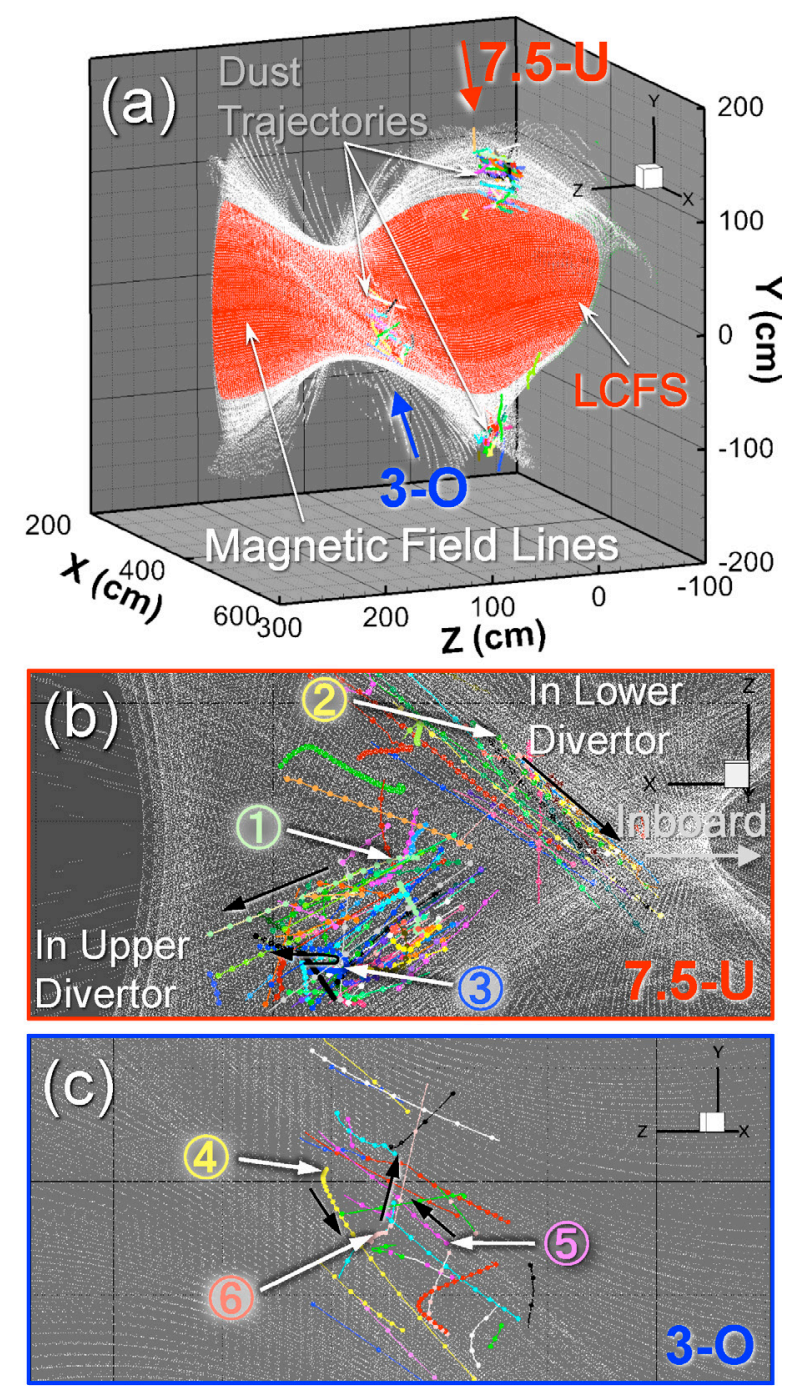

Figure 3 

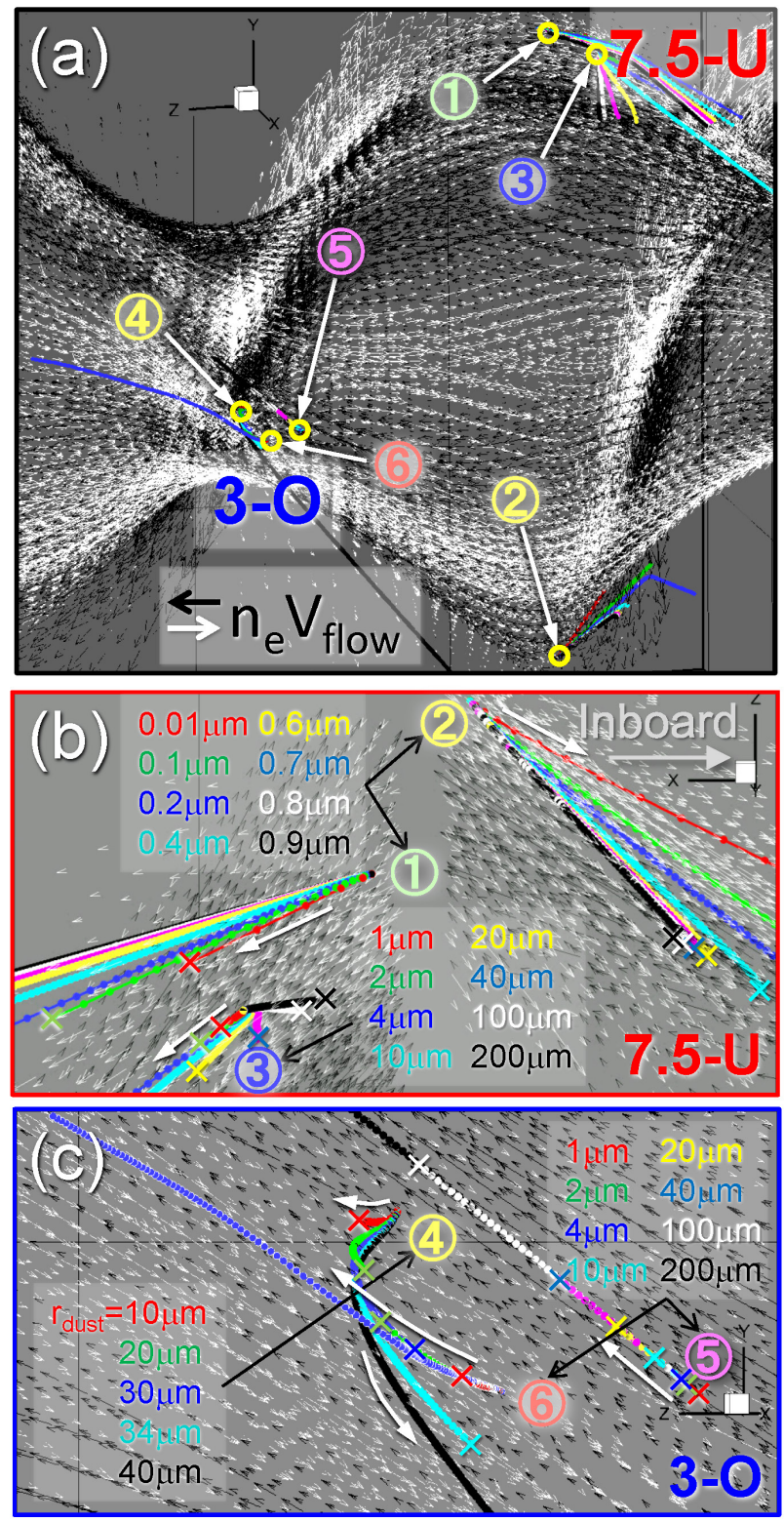

Figure 4 